\title{
INFLUENCE OF SURGICAL INTERVENTION ON PATIENTS WITH OVARIAN APOPLEXY IN THE ASPECT OF OVARIAN RESERVE PRESERVATION
}

\author{
O. M. Ishchak, \\ I. Horbachevsky Ternopil State Medical University \\ Ternopil Municipal Communal Clinical Hospital nr. 2, Ukraine
}

DOI: https://doi.org/10.31435/rsglobal_ws/31052019/6512

\section{ARTICLE INFO \\ Received: 10 March 2019 \\ Accepted: 11 May 2019 \\ Published: 31 May 2019}

\section{KEYWORDS}

ovarian apoplexy, ovarian reserve, Anti-Mullerian hormone (AMH), surgeries on adnexa.

\begin{abstract}
Ovarian apoplexy is the women's disease of reproductive age, $75 \%$ of which are under the age of 30 years old, about $30 \%$ undergo surgery, and $40-62 \%$ of them are subjected to repeated surgeries because of disease recurrence $[3,10]$.

Goal of study: improvement of surgical treatment of the patients with ovarian apoplexy for maximal preservation of ovarian reserve.

Materials and methods. A total of 120 women were included in the study. These include 55 patients with ovarian apoplexy, who underwent laparoscopic surgery for the first time. These patients were included in the prospective study and divided into two groups, depending on the hemostasis method used during the surgery. Group 1 included 30 patients with hemostasis, which was carried out using bipolar coagulation, group 2 included 25 patients, whose bleeding from the ovary was stopped by applying sutures. In each group, the patients were selected with the "blind" method. The study included patients with stable hemodynamic parameters before surgery and duration of hospitalization of not more than 24 hours. The comparison group consisted of 45 patients with laparoscopic confirmation of pain form of the ovarian apoplexy, who underwent conservative treatment. Study results and their discussion. Patients with ovarian apoplexy with stable hemodynamic parameters should undergo surgery using laparoscopic access, which allows to clarify the diagnosis, stop bleeding, prevent development of sexually transmitted infections and carry out surgical correction of the associated pathological processes of the internal genital organs. Stop of bleeding at the patients with ovarian apoplexy with bipolar electrosurgery is faster (shorter duration of surgery by 13 minutes), but this leads to more significant decrease in ovarian reserve (Anti-Mullerian hormone $(\mathrm{AMH})$ by $30 \%$, antral follicle count (AFC) by $10 \%)$ than use of hemostatic sutures (AMH by $22 \%$, PAF by $8 \%$ ) ( $\mathrm{p}<0.05)$. In order to maximize the maintenance of the ovarian reserve at surgeries by women with ovarian apoplexy, it is always better to carry out hemostasis by enucleating the cyst capsule within healthy tissues and saturation with absorbable synthetic areactive sutures on the ovarian wound following by intracorporal knot tying. Regardless of the hemostasis, used during surgical treatment of ovarian apoplexy by patients of the age of 36 years old and older, there is a greater decrease in ovarian reserve $\mathrm{AMH}$ by $30 \%, \mathrm{AFC}$ by $20 \%$ ) than at patients whose age is less than 35 years old (AMH by $20 \%$, AFC by $12 \%)(\mathrm{p}<0.05)$. By patients with implemented fertility function regardless of age and by all patients under the age of 35 , undergoing surgery because of ovarian apoplexy for the first time, bipolar coagulation is permissible to stop bleeding. At implementation of bipolar hemostasis of patients with ovarian apoplexy, point coagulation of bleeding vessels should be performed, avoiding damage to surrounding tissues.
\end{abstract}

Citation: O. M. Ishchak. (2019) Influence of Surgical Intervention on Patients with Ovarian Apoplexy in the Aspect of Ovarian Reserve Preservation. World Science. 5(45), Vol.2. doi: 10.31435/rsglobal_ws/31052019/6512

Copyright: (C) 2019 O. M. Ishchak. This is an open-access article distributed under the terms of the Creative Commons Attribution License (CC BY). The use, distribution or reproduction in other forums is permitted, provided the original author(s) or licensor are credited and that the original publication in this journal is cited, in accordance with accepted academic practice. No use, distribution or reproduction is permitted which does not comply with these terms. 
Introduction. Ovarian apoplexy is sudden hemorrhage into the ovary, accompanied by violation of the tissues integrity. There are two forms of apoplexy: algesic and hemorrhagic (anemic). In hemorrhagic case this pathological process is accompanied by bleeding into the abdominal cavity as a result of rupture of the vessels of the Graafian follicle, ovarian stroma, yellow body, corpus luteum cyst or follicular cysts. In the International Classification of Diseases of the WHO (ICD 10), the ovarian apoplexy corresponds to two nosological units: N83.0 - haemorrhagic follicular cysts of the ovary and N83.1 - hemorrhagic corpus luteum cyst. Frequency of ovarian apoplexy among all gynaecological diseases varies from $0.3 \%$ to $3 \%$ [10]. In the structure of acute gynaecological pathology apoplexy takes the third place (after ectopic pregnancy and acute inflammatory diseases of the uterine adnexa) - about $18 \%$, and the second (after ectopic pregnancy) in the structure of the causes of intraabdominal bleeding, although its share does not exceed $2.5 \%$ [4 7, 8]. Disease recurrence, according to the literature, is observed by $40-62 \%$ of patients $[7,8,9]$.

It is known that woman reproductive system is limited by a rather tight period of time and by the end of the third decade of life the process of loss of primary ovarian follicle pool, which peak is observed at the age of approximately thirty-seven years old, begins. Women of older reproductive age have significantly less impregnation ability in comparison to the patients of active reproductive age, which is associated with deterioration of the oocytes quality, violation of the endometrium receptor capacity, as well as the negative effects of concomitant or previous gynaecological and extragenital pathology [7]. By large part of the patients, suffering from infertility, use of standard protocols of assisted reproductive treatment is often ineffective, and they require use of donor oocytes [49, 80]. In addition to the natural loss of ovarian reserve with age increment of a woman, surgery, carried out on ovaries, performed in reproductive age, accompanied by loss of some tissue volume of one or both ovaries, also leads to its lowering. The issue of ovarian reserve reduction by infertile patients, who had previously undergone ovarian surgeries, arose in connection with the "poor response" to stimulating ovarian function in assisted reproductive treatment $[9,10]$.

In order to select patients with infertility for assisted reproductive treatment at the late 80's of the previous century, the concept of ovarian reserve was introduced [8]. Ovarian reserve is the number of primary ovarian follicles, apparently at a given time, is reflection of woman's reproductive age $[8,9]$.

To evaluate ovarian reserve, there was proposed study of a number of different biological factors: basal levels of follicle stimulating hormone (FSH), inhibin B, Anti-Mullerian hormone (AMH) as well as volume of ovaries, antral follicle count (AFC) and blood flow in the ovary artery during different phases of the menstrual cycle using transvaginal ultrasound examination and Doppler mapping [5]. To date, the most reliable criteria are the age of the patient, the level of AMH in the blood serum and transvaginal ultrasound, which is evaluated using transvaginal ultrasound examination $[4,5]$.

The main reason for reduction of ovarian reserve at enucleating of endometrioid cysts and benign ovarian tumors is the removal of part of healthy tissue together with the capsule that is confirmed histologically $[2,6,9]$. Some authors indicate that the amount of ovarian tissue removed directly dependents on the surgeon's qualification [1]. However, during multicenter studies, conducted with the participation of surgeons of expert level, it was found that even in the course of surgery by specialists of such qualifications similar tissue losses still occur [6,7].

Laparoscopic access, nowadays, is a "gold standard" in surgical treatment of the uterine adnexa pathology, including ovarian apoplexy, provides for the widespread use as hemostasis of bipolar coagulation. The action of electrosurgery is based on the rapid transition of electrical energy into heat one, spread of which from the point of impact on the surrounding healthy tissue leads to their damage. Necrosis, scar formation and disturbance of blood supply due to this damage can also lead to decrease of ovarian reserve. The present data on reduction of the ovarian reserve after cystectomy using bipolar coagulation and hemostatic sutures are controversial and require further study $[5,8,9]$.

Another potential cause of ovarian reserve reduction can be widespread use of electrosurgery during laparoscopic surgeries in order to provide hemostasis. The action of electrosurgery is based on the rapid transition of electrical energy into heat one, spread of which from the point of impact on the surrounding healthy tissue leads to their damage. Necrosis, scar formation and disturbance of blood supply due to this damage can also have negative effect on the functioning of follicular apparatus. However, data available to date on the comparative reduction of ovarian reserve after cystectomy using bipolar coagulation and hemostatic sutures are controversial. So in the projects, comparing the results of the endometriumctomy by laparoscopic surgery using bipolar hemostasis and similar laparotomic interventions, in which hemostasis was performed by suturing, significantly more reduction of ovarian reserve (AMH, AFC, FSH) was detected in the laparoscopic group $[4,5,10]$. Similar data on the AFC 
reduction after the application of bipolar coagulation in comparison with suturing are also reported in the preliminary report on laparoscopic removal of endometriumctomy [5]. In other randomized studies, comparing the effect of laparoscopic cystectomy on the ovarian reserve, no statistically significant differences between patients, underwent surgeries using bipolar coagulation and the patients, whose hemostasis was performed by intracorporal suture application were found [6, 7].

Equally contradictory data were obtained during studies of the status of ovarian reserve by patients with polycystic ovary syndrome after carrying out laparoscopic wedge resection: "Ovarian diathermy" or "Drilling" with monopolar electrosurgery. A number of researchers found a significant reduction in inhibin $\mathrm{B}, \mathrm{AMH}$ and $\mathrm{AFC}$, especially after bilateral surgeries $[5,6,7]$. However, some of them, during further study of the problem, noted that $\mathrm{AMH}$, reduced during the first week after the surgery, is restored to $65 \%$ of the pre-surgical level within 1-3 months [6]. Other studies did not reveal any changes in AMH and AFC after surgery [7, 8].

The state of the ovarian reserve by patients with ovarian apoplexy is practically not investigated. One of the tasks of the thesis is to study the reproductive health aspects of women, who suffered from this disease, statistically significant increase in basal level of FSH and decrease of AFC by $36.8 \%$ of patients after repeated apoplexy [2].

This state means necessity to study the indicators of ovarian reserve with this disease by patients underwent surgical treatment.

\section{Goal of study:}

- improvement of surgical treatment of the patients with ovarian apoplexy for maximal preservation of ovarian reserve.

\section{Materials and methods.}

A total of 120 women were included in the study. These include 55 patients with ovarian apoplexy, who underwent laparoscopic surgery for the first time. These patients were included in the prospective study and divided into two groups, depending on the hemostasis method used during the surgery. Group 1 included 30 patients with hemostasis, which was carried out using bipolar coagulation, group 2 included 25 patients, whose bleeding from the ovary was stopped by applying sutures. In each group, the patients were selected with the "blind" method. The study included patients with stable hemodynamic parameters before surgery and duration of hospitalization of not more than 24 hours. All surgeries were performed by the same surgical team. Criteria for exclusion from the study were bleeding into the abdominal cavity as a result of the breakdown of benign tumor or endometriotic ovarian cyst; presence in the history of surgical interventions on the ovaries; as well as the use of combined oral contraceptives or the use of multifollicular ovarian stimulation methods with exogenous gonadotropins less than 3 months before surgery. The comparison group consisted of 45 patients with laparoscopic confirmation of pain form of the ovarian apoplexy, who underwent conservative treatment.

Surgery began with the detection of the source of bleeding and performing hemostasis. In cases of frank adhesive process, which did not allow identifying the source of bleeding visually, adhesion to the extent necessary for hemostasis was initially performed. In group 1, the bleeding was stopped by the point effect of bipolar instrument with $3 \mathrm{~mm}$ width jaw of directly to the vessel, which current power was $35 \mathrm{~W}$. In case of ineffectiveness of point coagulation, the cyst capsule was enucleated with subsequent additional point hemostasis of the bleeding sites in the cyst area. In group 2 , in all cases, to stop bleeding, cysts were enucleated and 1-3 separate sutures number $5 / 0$ with polyglycolic acid absorbable threat were applied on the ovarian wound followed by intracorporeal knot tying technique. After the end of hemostasis lavage of the abdominal cavity was performed with physiological solution. To remove blood clots and liquids from the upper floors of the abdominal cavity, the patient was transferred to the Fowler's position (30 degrees).

The magnitude of post-surgical bleeding was determined by counting the difference between the volume of fluid introduced into the abdominal cavity and the volume of fluid in the vacuum suction cup after the surgery.

The reproductive age of a woman is an ovarian reserve: existing primary ovarian follicles presently available, capable of providing oocyte maturation and fertilization [6,8]. The markers of the ovarian reserve are age of the patient, the level of Anti-Mullerian hormone (AMH) in blood serum and antral follicle count (AFC), calculated by transvaginal ultrasound examination $[1,2,5]$.

\section{Study results and their discussion:}

Patients with ovarian apoplexy with stable hemodynamic parameters should undergo surgery using laparoscopic access, which allows to clarify the diagnosis, stop bleeding, prevent development 
of sexually transmitted infections and carry out surgical correction of the associated pathological processes of the internal genital organs. Stop of bleeding at the patients with ovarian apoplexy with bipolar electrosurgery is faster (shorter duration of surgery by 13 minutes), but this leads to more significant decrease in ovarian reserve (Anti-Mullerian hormone (AMH) by $30 \%$, antral follicle count (AFC) by $10 \%)$ than use of hemostatic sutures (AMH by $22 \%$, PAF by $8 \%)(\mathrm{p}<0.05)$. Patients with ovarian apoplexy should be informed of probable loss of the ovarian reserve as a result of surgical treatment and patients over the age of 36 years old with unrealized generative function, undergoing repeated surgical intervention, should be warned that these losses can cause infertility.

In order to maximize the maintenance of the ovarian reserve at surgeries by women with ovarian apoplexy, it is always better to carry out hemostasis by enucleating the cyst capsule within healthy tissues and saturation with absorbable synthetic areactive sutures on the ovarian wound following by intracorporal knot tying. Regardless of the hemostasis, used during surgical treatment of ovarian apoplexy by patients of the age of 36 years old and older, there is a greater decrease in ovarian reserve (AMH by 30\%, AFC by 20\%) than at patients whose age is less than 35 years old (AMH by $20 \%$, AFC by $12 \%$ ) ( $<<0.05)$. By patients with implemented fertility function regardless of age and by all patients under the age of 35, undergoing surgery because of ovarian apoplexy for the first time, bipolar coagulation is permissible to stop bleeding. At implementation of bipolar hemostasis of patients with ovarian apoplexy, point coagulation of bleeding vessels should be performed, avoiding damage to surrounding tissues. By patients with ovarian apoplexy with unrealized reproductive function of older reproductive age (36 years and older) and patients undergoing repeated surgical intervention, regardless of age, preferably refuse to use bipolar hemostasis and only to apply sutures to stop bleeding. At women treated because of ovarian apoplexy twice, there is greater reduction of ovarian reserve (AMH by $40.6 \%, \mathrm{AFC}$ by $28 \%$ ) than underwent surgery once. The ovarian reserve decreases to critical values with an increase in the number of surgeries (over 2) for ovarian apoplexy (AMH by $55 \%$, AFC by $30 \%$ ) and women's age (36 years and older) (AMH by $60 \%$, AFC by $40 \%$ ) (p $<0.001$ ). For patients with ovarian apoplexy, who are not planning childbirth shortly after surgery, there should be recommended combined oral contraceptive or contraception and prophylaxis of ovarian apoplexy recurrence. By women over the age of 36, who have undergone 2 or more ovarian apoplexy surgeries that have not become pregnant 12 months with non-contraceptive sexual intercourse, a standard infertility test protocol should include AMH level study and AFC calculation.

\section{Conclusions.}

1. Ovarian apoplexy leads to decrease, rather small (AMH by $8 \%$, AFC by $9 \%$ ) of ovarian reserve $(\mathrm{p}>0.05)$.

2. Surgical treatment of patients with ovarian apoplexy leads to a significant continuous decrease in ovarian reserve during 6 menstrual cycles after surgery, regardless the hemostasis method used $(\mathrm{p}<0.05)$.

3. Reducing ovarian reserve by patients with ovarian apoplexy after a single surgical treatment, regardless of the hemostasis method, is not critical. AMH and AFC remain at high or average levels.

4. The prognostic factor of spontaneous pregnancy after surgery by patients with ovarian apoplexy may be age but not ovarian reserve index (AMH and AFC).

\section{Practical novelty.}

The results of study allowed determining the ways of improving the surgical treatment of patients with ovarian apoplexy from the position of maximal preservation of the ovarian reserve, as well as the follow-up tactics of these patients, depending on age, state of reproductive performance and the number of surgeries because of this disease. Implementation of the recommendations, developed in this study, will allow performing maximal preservation of ovarian reserve and reproductive function and thus improving the results of surgical treatment of patients with ovarian apoplexy. 